\title{
214. Sodium Dodecyl Sulfate and Tyrosyl Ionization in Bovine Serum Albumin
}

\author{
By Osamu Takenaka, Shiro Aizawa, Yoshihisa Nishimura, \\ Jun Hirano, and Yuji INADA \\ Lab. Biol. Chem., Tokyo Institute of Technology, Meguro-ku, Tokyo
}

(Comm. by Tanemoto Furuhata, M. J. A., Dec. 12, 1969)

Introduction. The serum albumin of mammals makes about half of the blood plasma protein, and plays an important role in the transport of a variety of substances in blood. Interesting behaviors in its conformational changes as well as the binding capacity of various compounds, ${ }^{1)}$,2) have long been attracting biochemical and physicochemical investigators of proteins.

In recent years many methods for elucidating the tertiary structure of proteins have been developed. ${ }^{3)-5}$ ) We have hoped that the application of these methods might cast some new light on the complicated problem of the structure and function of serum albumin.

The present paper is concerned with the analysis of the ionization of tyrosyl residues in bovine serum albumin (BSA) and the suppressive effect of sodium dodecyl sulfate (SDS) and that of a series of fatty acids on tyrosyl ionization.

Materials and method. The crystalline BSA purchased from the Nutritional Biochemicals Corp. was defatted with charcoal according to the method described by Sogami and Foster, ${ }^{6}$ ) which is a slight modification of the one developed by Chen. ${ }^{7}$ ) The defatted BSA was kept in a solution of ionic strength 0.05 and $\mathrm{pH} 5.6$ at $3^{\circ} \mathrm{C}$. The denatured BSA was obtained by contact with alkali solution of $\mathrm{pH}$ 13.1 for 7 hours at $4^{\circ} \mathrm{C}$. The molar extinction coefficient $(\varepsilon)$ of BSA at $279 \mathrm{~m} \mu$ was assumed to be $4.4 \times 10^{4} \mathrm{~mole}^{-1} \mathrm{~cm}^{-1}{ }^{8)}$ Ionization curves of tyrosyl residues of BSA were obtained spectrophotometrically in the following manner. A mixture $(3.0 \mathrm{ml})$ of $0.2 \mathrm{~N} \mathrm{KOH}$ and $0.2 \mathrm{~N} \mathrm{KCl}$ solutions was added to an aqueous solution of BSA $(1.0 \mathrm{ml})$. The volume ratio between these $\mathrm{KOH}$ and $\mathrm{KCl}$ solutions was varied so as to obtain an alkaline BSA solution of varied $\mathrm{pH}$ at a fixed ionic strength, 0.15. In an experiment for BSA in the presence of various concentrations of SDS or fatty acids, $\mathrm{Na}_{2} \mathrm{HPO}_{4}-\mathrm{KOH}$ buffer (pH-11.8-11.9) of ionic strength of 0.20 was used instead of the $\mathrm{KOH} \mathrm{KCl} \mathrm{mixture.} \mathrm{The} \mathrm{difference,} \Delta E$, in absorbance at $295 \mathrm{~m} \mu$ between this alkaline BSA solution and the neutral BSA solution at the same protein concentration and the same ionic strength was 
measured at $18^{\circ} \mathrm{C}$ with a Shimadzu recording spectrophotometer model SV-50, using $1.0-\mathrm{cm}$ cells. The $\Delta E$ and $\mathrm{pH}$ values were read immediately after preparation of the alkaline BSA solution. $\Delta E$ values were converted to the molar concentrations of ionized tyrosine with aid of $\Delta \varepsilon=2,300,{ }^{9)}$ the difference in molar extinction coefficient at $295 \mathrm{~m} \mu$ between ionized and non-ionized forms of tyrosine. Throughout this paper, the moles of hydroxyl ion involved in the ionization of tyrosyl residues per mole of tyrosine is expresised by $m$, and the moles of tyrosyl residues of the same type per mole of BSA by $n$.

Results, 1) Native BSA. The ionization curve of tyrosyl residues of $1.64 \times 10^{-5} \mathrm{M}$ BSA is shown by curve A in Fig. 1 . This curve tends to approach the constant level, $n=20$, which agrees with the molar content of tyrosine determined by amino acid analysis. ${ }^{8)}$ The analysis of this curve was undertaken according to the procedures described by Inada.9),10) Curve A below pH 11.0 is found to fit a theoretical curve with $\mathrm{pK}=\mathbf{1 0 . 9}$ and $m=1.0$ (curve $\mathrm{B}$ ) and the curve $\mathrm{B}$ approaches the constant level of $n=4.0$. Curve $\mathrm{C}$ was obtained by subtracting curve B from curve A. Analysis of this curve showed that the curve is a composite of theoretical ionization curves of two types of tyrosyl residues; ten residues with $\mathrm{pK}=\mathbf{1 1 . 5}$ and $m=3.0$ (curve D) and six residues with $\mathrm{pK}=12.3$ and $m=2.0$ (curve $\mathrm{E})$.

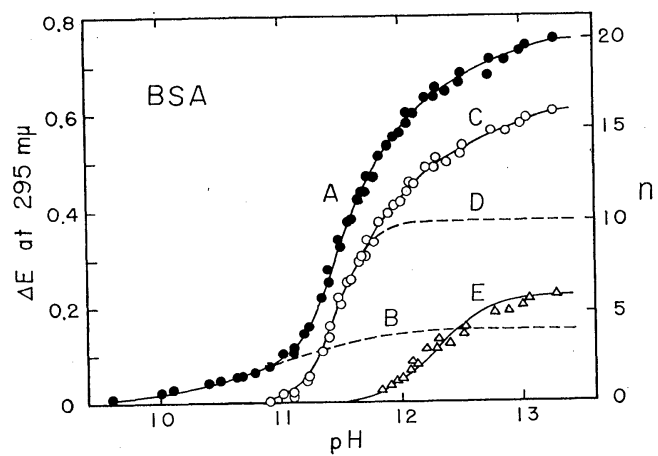

Fig. 1. Ionization curves of tyrosyl residues in $16.4 \mu \mathrm{M}$ BSA: curve A, observed values after preparation of alkaline sample solution, curves $\mathrm{B}, \mathrm{D}$ and $\mathrm{E}$ are the theoretical ionization curves obtained by calculation for 4 residues with $\mathrm{pK}=10.9(m=1.0), 10$ residues with $\mathrm{pK}=11.5$ $(m=3.0)$ and 6 residues with $\mathrm{pK}=12.3(m=2.0)$, respectively. The $\triangle \mathrm{E}$ values shown by $\bigcirc-\bigcirc$ on curve $C$ were obtained by subtracting the theoretical values for the residues with $\mathrm{pK}=10.9$ (curve $\mathrm{B}$ ) from the observed values on curve $A$. The values shown by $\triangle-\triangle$ on curve $E$ were obtained by subtracting the sums of the values calculated for the residues with $\mathrm{pK}=10.9$ and 11.5 (curves $\mathrm{B}$ and $\mathrm{D}$ ) from the observed values on curve $\mathrm{A}$. 
2) Native BSA in the presence of SDS. The ionization curve of tyrosyl residues of $\mathrm{BSA}$ in the presence of SDS is shown by curve A in Fig. 2. The same analysis as described above was worked on the curve and yielded curves B, C, D and E. The tyrosyl residues of the BSA molecule in the presence of SDS are grouped into three types; four residues with $\mathrm{pK}=11.3$ and $m=1.0$ (curve $\mathrm{B}$ ) ten with $\mathrm{pK}=11.9$ and $m=5.0$ (curve $\mathrm{D}$ ) and the remaining six with $\mathrm{pK}=12.5$ and $m=3.0$ (curve E). The inserted figure in Fig. 3 shows the ionization curves of BSA in the presence (curve B) and absence (curve A) of SDS. Curve $C$ in the insert obtained by subtracting curve $B$ from curve $\mathrm{A}$ represents the $\mathrm{pH}$-dependency of the depression of tyrosyl ionization due to the presence of SDS. As seen from Fig. 3, the

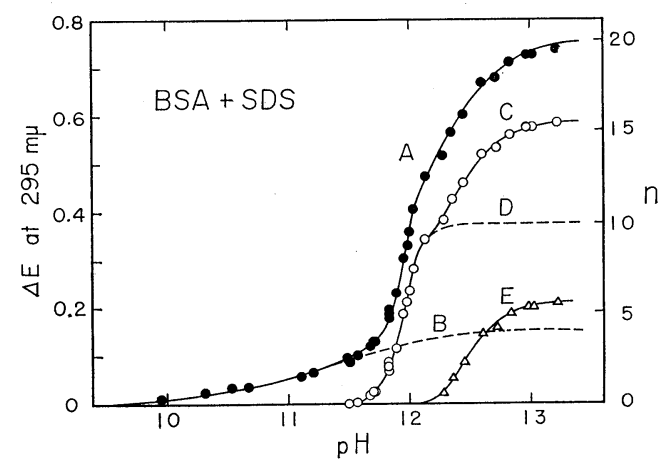

Fig. 2. Ionization curves of tyrosyl residues in $16.4 \mu \mathrm{M}$ BSA: Curve A, observed values after preparation of alkaline sample solution, curves B, D and $\mathrm{E}$ are the theoretical ionization curves obtained by calculation for 4 residues with $\mathrm{pK}=11.3(m=1.0), 10$ residues with $\mathrm{pK}=11.9$ $(m=5.0)$ and 6 residues with $\mathrm{pK}=12.5(m=3.0)$, respectively. The $\triangle \mathbf{E}$ values shown by $\bigcirc-\bigcirc$ on curve $C$ were obtained by subtracting the theoretical values for the residues with $\mathrm{pK}=\mathbf{1 1 . 3}$ (curve $\mathrm{B}$ ) from the observed values on curve $A$. The values shown by $\triangle-\triangle$ on curve $\mathbf{E}$ were obtained by subtracting the sums of the values calculated for the residues with $\mathrm{pK}=\mathbf{1 1 . 3}$ and 11.9 (curves $\mathrm{B}$ and $\mathrm{D}$ ) from the observed values on curve $A$.

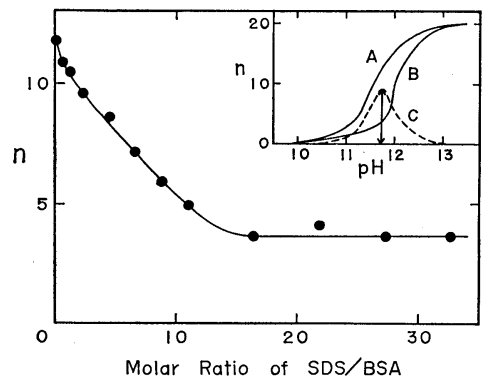

Fig. 3. Suppression of tyrosyl ionization in BSA in the presence of SDS: Curves $A$ and $B$, the ionization curves of tyrosyl ionization of $16.4 \mu \mathrm{M}$ BSA in the absence and presence of $240 \mu \mathrm{M}$ SDS, respectively. Curve $\mathrm{C}$, obtained by subtracting curve $\mathrm{B}$ from curve A. 
number of ionized tyrosyl residues decreases with increasing the concentration of $\mathrm{SDS}$, and at the molar ratio of $\mathrm{SDS} / \mathrm{BSA}=15$ the ionization of nine residues is disturbed by SDS.

3) Alkali-denatured BSA with and without SDS. The ionization curves of alkali-denatured BSA in the absence and presence of SDS run nearly along the same trail, as shown by the solid and open circles in Fig. 4. The analysis of the curve(s) revealed only two types of tyrosyl residues; four residues with $\mathrm{pK}=9.9$ and $m=1.0$, which agree with those of free tyrosine ${ }^{9)}$ and the remaining 16 residues with $\mathrm{pK}=11.1$ and $m=1.0$. The ionization characteristics of BSA and alkali-denatured BSA in the absence and presence of SDS are summarized below.

4) Effect of fatty acids. BSA has a strong affinity to fatty acids. It may be of some interest to see if there is any relationship between the chain length of fatty acids and their suppressive effect on tyrosyl ionization. The result is shown in Fig. 5, in which the ordinate is expressed by the number of tyrosyl residues suppressed in the presence of fatty acids. Curves $A$ and $B$ represent the results obtained for two different molar ratios of fatty acid/BSA, 9.0 and 4.5 , respectively. It is interesting that there is a maximal suppres-

Table I

\begin{tabular}{rccrccrrr}
\hline & BSA & \multicolumn{3}{c}{ BSA+SDS } & \multicolumn{3}{c}{ Denat's BSA } & \multicolumn{1}{c}{ BDS } \\
\hline$n$ & $m$ & $\mathrm{pK}$ & $n$ & $m$ & $\mathrm{pK}$ & $n$ & $m$ & $\mathrm{pK}$ \\
\hline 4 & 1 & 10.9 & 4 & 1 & 11.3 & 4 & 1 & 9.9 \\
10 & 3 & 11.5 & 10 & 5 & 11.9 & 16 & 1 & 11.1 \\
6 & 2 & 12.8 & 6 & 3 & 12.5 & & & \\
\hline
\end{tabular}

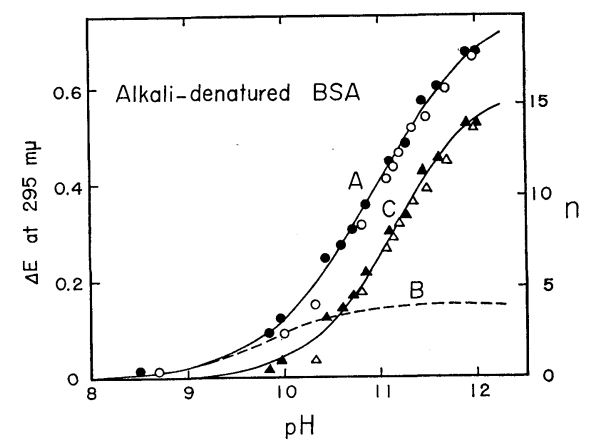

Fig. 4. Ionization curves of tyrosyl residues in $16.4 \mu \mathrm{M}$ alkali-denatured BSA in the absence and in the presence of $240 \mu \mathrm{M}$ SDS; The values shown by $-O$ and $\bigcirc$ on curve A are observed values without and with SDS, respectively. Curves $B$ and $C$ are the theoretical ionization curves obtained by calculation for 4 residues with $\mathrm{pK}=9.9(m=1.0)$ and 16 residues with $\mathrm{pK}=11.1 \quad(m=1.0)$. The values shown by $\Delta-\Delta$ and $\triangle-\triangle$ on curve $\mathrm{C}$ were obtained by subtracting the values calculated for 4 residues with $\mathrm{pK}=9.9$ (curve $\mathrm{B}$ ) from the observed values on curve $\mathrm{A}$. 


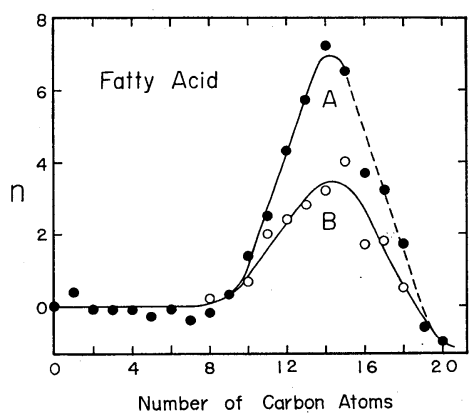

Fig. 5. Suppression of tyrosyl ionization in $18.1 \mu \mathrm{M}$ BSA in relation to the number of carbon atoms of fatty acids. The values shown by on curve $A$ are the observed values at the concentration of fatty acid, $160 \mu \mathrm{M}$, and $\bigcirc-\bigcirc$ are that of $80 \mu \mathrm{M}$. In the range shown by the broken line of curve A, optical measurements were slightly disturbed by weak opalescence in the solution. The errors due to it were eliminated by subtracting the increased optical attenuance at $340 \mathrm{~m} \mu$ from the observed value of $\triangle \mathrm{E}$ at $295 \mathrm{~m} \mu$.

sive effect at a certain chain length, $\mathrm{C}=14,15$.

Discussion. Tyrosyl ionization in human and bovine serum albumins with or without fatty acids and detergents has been investgated by many workers. ${ }^{11)-14)}$ Most of these works were carried out in connection with either the physicochemical treatment of the titration behaviors of albumins or with the estimation of the binding sites in the molecule.

The first 4 residues ionized in BSA, BSA with SDS, and denatured BSA with and without SDS, have the same $n$ and different pK-values, suggesting that the ionization properties of the 4 residues located on the surface or near the surface of the BSA molecule are changed, if any, slightly by the binding of SDS or by the alkalidenaturation. In contrast, the ionization properties of the 10 residues of the second group are changed significantly by the presence of SDS. The $m$ value of these residues is changed from 3.0 to 5.0 and the pK value from 11.5 to 11.9 by the presence of SDS. This may indicate that the ten tyrosyl residues have a closets relation with the binding capacity of BSA for SDS. The curve $C$ in the insert of Fig. 3 indicates that at least 9 tyrosyl residues are transformed into less ionizable form by the presence of SDS. The remaining six residues of the third group in BSA have abnormally high pK values both in the absence and presence of SDS and may be embedded in the interior of the BSA molecule. The ionization properties of sixteen residues ( $\mathrm{pK}=11.1$ and $m=1.0$ ) in the denatured BSA do not change by the addition of SDS, indicating an inactivation of the binding sites of BSA. BSA, a globular protein, was supposed to consist of three subunits connected by single peptide chain ${ }^{15)}$ and to expand at $\mathrm{pH} 11.3$, 
and, this expansion is suppressed in the presence of SDS. ${ }^{11}$ The suppression of the expansion may be reflected on the elevation of the $\mathrm{pK}$ value and of $m$ values from 3.0 to 5.0 and 2.0 to 3.0 in our experiments.

As for the denatured BSA, we shall refrain from detailed discussion, except that denaturation seems to make all the tyrosyl residues free, and that SDS does not seem to bind to the denatured BSA, or though less likely, if it binds, binding sites have little or no association with tyrosyl residues. In connecton with this, it should be noticed that the denaturation may have interfered with our ionization measurements at extremely high pH's.

Any definite conclusion with respect to the experiments on fatty acids may need further systematic investigations with, for example, many kinds of fatty acid derivatives, including branchedchain isomers.

Summary. Ionization of tyrosyl residues in charcoal-defatted bovine serum albumin was measured spectrophotometrically in the absence and presence of sodium dodecyl sulfate and 20 straight chain fatty acids. Analysis of the experimental data revealed at least three distinct groups of tyrosyl residues in BSA, each including 4, 10 and 6 residues. Suppressive effect of SDS and fatty acids on the tyrosyl ionization has been confirmed.

Acknowledgement. The authors are grateful to Dr. T. Komai for his kind encouragement in carrying out this work.

\section{References}

1) Putnam, F. W. (1965): In The Proteins (Ed. H. Neurath). Acad. Press, New York, N.Y. Vol. 3, 187pp.

2) Sogami, M. (1969): Plasma Albumin (in Japanese): Kagaku-no-Ryo-iki, 23, 512-520; 602-609.

3) Timasheff, S. N., and M. J. Gornbunoff (1967): Ann. Rev. Biochem., 36, 13-54.

4) Cohen, L. A. (1968): Ibid., 37, 695-726.

5) Shibata, K. (1969): Chemical Investigation Method of Protein Conformation (in Japanese). Univ. Tokyo Press, Tokyo, Japan.

6) Sogami, M., and J. F. Foster (1968): Biochemistry, 7, 2172-2182.

7) Chen, R. F. (1967): J. Biol. Chem., 242, 173-181.

8) Spahr, P. F., and J. T. Edsall (1964): Ibid., 239, 850-854.

9) Inada, Y. (1961): J. Biochem. Tokyo, 49, 217-225.

10) Inada, Y., M. Kamata, A. Matsushima, and K. Shibata (1964): Biochim. Biophys. Acta, 81, 323-335.

11) Lovrien, R. (1963): J. Am. Chem. Soc., 85, 3677-3682.

12) Decker, R. V., and J. F. Foster (1967): J. Biol. Chem., 242, 1526-1532.

13) Reynolds, J., S. Herbert, and J. Steinhardt (1968): Biochemistry, 7, 13571361.

14) Zakrzewski, K., and H. Goch (1968): Ibid., 7, 1835-1842.

15) Bloomfield, V. (1966): Ibid., 5, 684-689. 